\title{
Device and Method for Precise Repositioning of Subjects for 3D Imaging of Head, Face, and Neck
}

\author{
Richard C. ROTH*, Matthew DEPAUW, Andrew HEPNER \\ Advanced Imaging and Measurement Laboratory, Amway Corporation, Ada (MI), USA
}

\begin{abstract}
Three dimensional imaging has been made possible through the development of laser and white light interference fringe technology. These technologies have allowed subjects to directly view improvements in facial contours due to cosmetic product applications and dermatologic procedures. Laser based scanning has the drawback of the need for closed eyes; white light interference fringe scanning requires very low light backgrounds and employs short bursts of relatively bright light. Both technologies require subjects to remain extremely still. Differential comparison of 3D images, as image overlays, not only exacerbate the need for still subjects, but also the need for precise alignment of the subjects in order to observe small structural and contour changes to the head, face and neck regions. These changes are observed as a result of efficacious cosmetic product applications and invasive dermatological cosmetic procedures for anti-aging, sagging, and lifting interventions, and in weight loss.
\end{abstract}

Subject orientation without encumbrances of the face or neck, and without distortion of facial features, is a special concern with $3 \mathrm{D}$ imaging. Stillness of the subject is a concern, but co-location of the subject for subsequent imaging sessions is paramount for high quality 3D image overlay. The positioning devices presented provide for a novel set of alignment processes including, gross subject alignment, fine subject alignment, and direction-of-gaze. Gross alignment is accomplished through defined general positioning of body and head, fine positioning includes subject specific head alignment, and critically, the subject direction-of-gaze during head alignment. Parameters of the positioning device are recorded during an initial "subject fitting" and define a subject-specific orientation for subsequent imaging sessions.

The positioning device provides unencumbered imaging of the subject facial regions and a return to the same orientation according to preset subject specific fitting parameters. Contact with head touch points are maintained by the subject and allow undistorted full facial imaging and analysis of the face, jaw, neck regions. The touch points are defined by the height above the seated position, distance from vertical orientation, occipital head location, and a top frontal position which combines the subjects head curvature and size. These defined parameters are combined with a specific directionof-gaze. Through the positioning device and alignment process the four axes of co-location: depth, tilt, rotation, and yaw, are addressed. Re-alignment parameters are maintained within 0.1 to $0.2 \mathrm{~mm}$ for all reticulated adjustments.

In this presentation, a set of integrated positioning devices is described which, 1) align the subject body and head, 2) co-locate the head and neck for repeatable analysis, and 3) maintain the directionof-gaze of the subject. Comparisons are observed as measured differences in horizontal and vertical cross-sections, color-coded 3D contour maps, and as regional distance and volume changes. Three dimensional images illustrating overlay, cross-sectional analysis, contour, and volume changes from applications and procedures are presented.

Keywords: 3D, parallax, reposition, face

\section{Introduction}

Three-dimensional (3D) imaging has been made possible through the development of laser and white light interference fringe technology $[1,2]$. These technologies have allowed subjects to directly view improvements in their own facial contours as a result of cosmetic product applications and dermatologic procedures. Laser based scanning requires that subject always have closed eyes; where white light interference fringe scanning requires very low light backgrounds and employs short bursts of relatively bright light that may startle subjects. Both technologies require that the subject remain extremely still. Differential comparison of 3D images, as image overlays, not only exacerbate

\footnotetext{
* rick.roth@amway.com; 1-616-787-5354; www.amway.com
} 
the need for still subjects, but require precisely aligned subject images in order to observe small structural and contour changes to the head, face and neck regions. Such changes are observed following efficacious cosmetic product applications; invasive dermatological procedures for anti-aging, sagging, and lifting interventions; and weight loss.

A number of methods have been suggested and are in use for positioning subject for facial imaging. These methods either do not take into account repositioning of the subject for equivalent images at a later date, or significantly restrict or deform the face, head, and neck regions accessible for imaging. Some include chin rests, ear bud restraints, and forehead restraints which provide solid reference points for the camera, but do not maintain subject position during realignment [3, 4]. Some methods have rests that obscure portions of the face and neck, or the angle of the subject's head and neck was not controlled with each analysis. The more precisely the subjects are aligned for each analysis the better it will be for subsequent image repositioning and visualization of small differences in contour over time.

The integrated positioning device described, 1) aligned the subject body and head, 2) co-located the head, face and neck region for repeatable analysis, and 3) maintained the subject direction-of-gaze [5]. Seating, body and head alignment and especially the direction-of-gaze protocol were critical and required subject education prior to image acquisition. Device adjustment parameters were recorded for each subject. Parameter measurement to within 0.1-0.2 mm allowed entire system reset for each subject. An alignment protocol is given and comparisons of images at different time points are reported as measured differences in horizontal and vertical cross-sections, color-coded 3D contour maps, and as regional distance and volume changes. Three-dimensional images illustrating overlay, cross-sectional analysis, contour, and volume change from applications and procedures are presented.

\section{Methods and Materials}

\subsection{Alignment principle}

The positioning devices described provide a novel set of alignment processes including, gross subject alignment, fine subject alignment, direction-of-gaze, and computer adjustment for image overlay. Gross alignment was accomplished through defined general positioning of body and head; fine positioning included centering, and leveling; and direction-of-gaze defined by subject-specific head alignment integrated the subject to the camera and sensor positions. Physical position parameters of the device were determined during initial "subject fittings" and defined subject-specific locations recorded for subsequent imaging sessions.

\subsection{Subject preparation and education}

At registration, the subjects were provided a fitted soft black v-cut blouse, black headband for pulling hair away from forehead and ears, and a black hair net to assure that stray hair was away from the face. The forehead and ears were visible for imaging and 3D alignment purposes. The use of soft black cloth for the blouse, hair band and hair net assisted in eliminating unnecessary imaging data.

The positioning device allowed unencumbered imaging of the facial regions and provided the means to reorient the subject using the series of subject-specific parameters obtained at the initial fitting. . In order to obtain unencumbered and co-located images, the device and alignment process were designed to minimize the majority of subject tilt, twist rotation, and depth variation.

Alignment process education (Fig. 1) was essential to reduce subject movement. Subject familiarity with the process of seating, alignment, and imaging improved the initial alignment efforts and greatly reduced time for subsequent subject positioning. Prior to imaging, the subjects viewed a short video presentation on the equipment and alignment process, and were able to review and manipulate a handheld demonstration model of the parallax bar system. The alignment process of seating, body positioning, and head positioning was described to each subject in the imaging room immediately prior to seating. The subject was then realigned three times (as practice) to improve subject understanding of the alignment process prior to image capture.. 


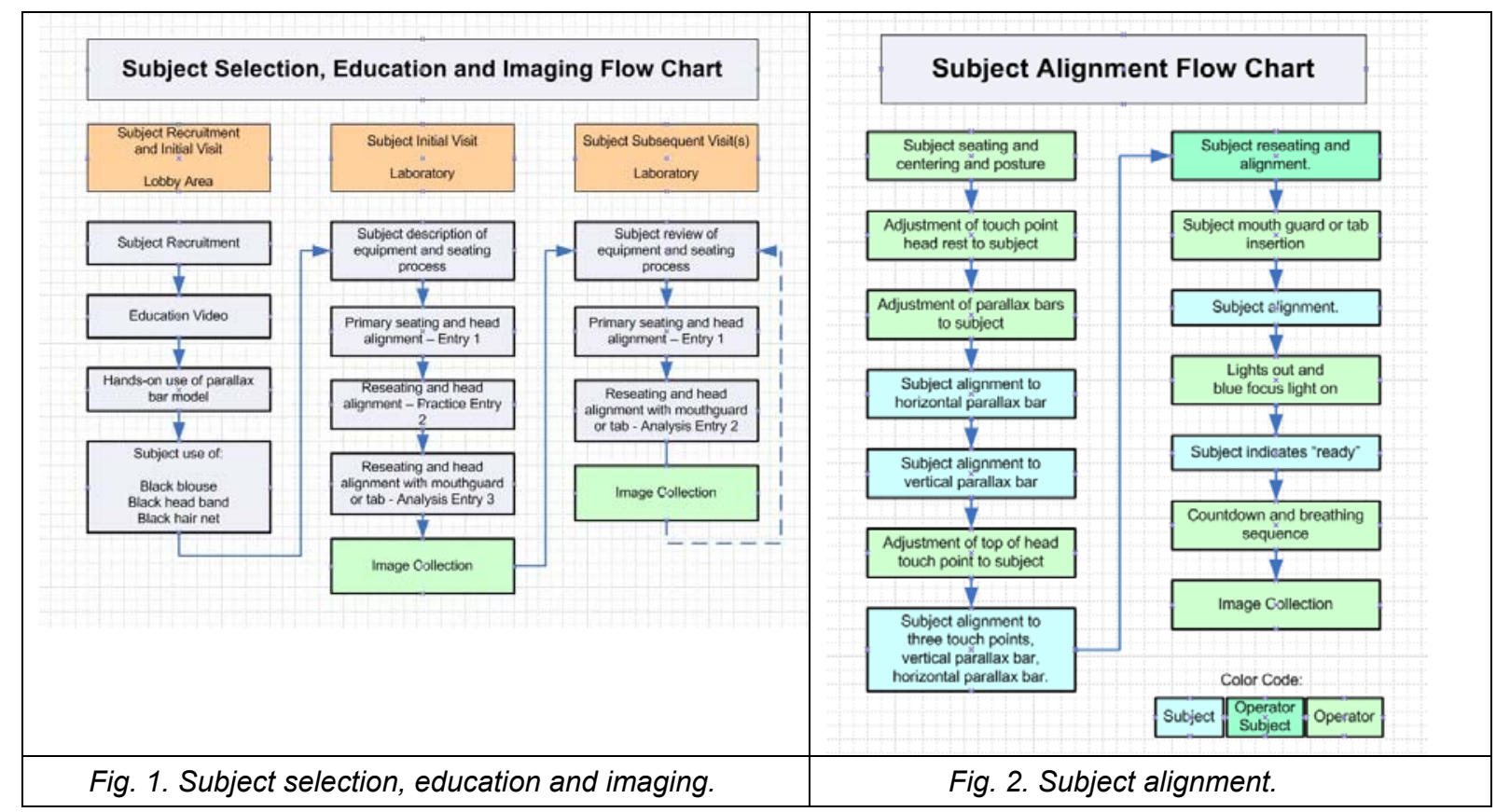

\subsection{Body Alignment}

Body alignment included seating and weight location, arm and leg placement and body centering (Fig. 2.). The subject faced the parallax bar and camera system with back to the chair and feet directly proximal to the chair foot rest. The subject sat, posterior first, and slid back with instructions to evenly and uniformly distribute their sitting weight. Feet were placed centrally and evenly on the footrest with balls of the feet at the edge of the footrest. Elbows were placed on the side arm rests, and the hand rest lowered. The hands are placed in front, centered, with fingers touching but not overlapping. The subject was checked for center positioning using the 2D camera love view and the live view of the 3D camera sensor system.

\subsection{Direction-of-Gaze and Parallax Bars Orientation Protocol}

The subject was seated erect and centered in the chair. The alignment stand height adjustment and headrest position were moved to a subject-specific position and locked for the direction-of-gaze facial orientation protocol. The subject maintained their head in contact with the two headrest touch points throughout the protocol. Tilt and twist were minimized using adjustable parallax bars mounted horizontally and parallel to each other within the parallax bar frame. The bar closer to the subject was a lighter color than the more remote bar. The subject used these bars as up-down tilt adjustment references. The subject directed their gaze forward at the two bars; the bar assembly was raised (or lowered) to meet the subject's horizontal sight plane. Once the bars were aligned at the direct horizontal view, the subject indicated to the operator to perform a fine adjustment to overlay the view of the bars. The subject obtained fine orientation through minor head left-right rotation such that the entire length of the lighter, closer, bar completely and evenly occluded the entire length of the more remote, darker, bar.

While maintaining the horizontal parallax bar overlay, the subject focused on the more remote of the two vertical parallax bars. Focus on the more distant vertical bar created binocular vision that visually produced two closer bars out of one. The closer bars bracket, left and right, the more distant vertical bar. The subject then performed an extremely minor left/right head adjustment to exactly center the more distant vertical bar between the closer visualized bars. Focus on the cross points of the vertical and horizontal bars and maintenance of this position held the direction-of-gaze and head orientation. The frontal bone touch point was adjusted to the subject and lowered onto the subject's head above the hair line. The frontal bone touch point provided the third alignment reference point. Subject alignment with the horizontal and vertical parallax bars was then re-confirmed. .A small neck alignment bar was brought into position to touch the back of the neck. The neck bar did not provide any support and only served as a reminder of neck position for subsequent alignments. 
The subject fully removed themself from the chair apparatus and conducted a reseating and realignment (with assistance for seating and centering from the operator). The realignment was conducted with all device parameters locked in place for the subject. With this action, the subject had undergone both full education of the alignment system and the re-seating alignment protocol. The subject then removed their head only, and a jaw restraint in the form of either a mouth freshening tab held between the teeth, or an individually fitted mouth-guard was inserted. The subject reentered the headrest and raised one index finger to indicate proper alignment.

With lights dimmed, the operator provided a countdown to the subject while the subject began to exhale. The 3D and 2D images were immediately obtained. Optional images at adjustable chair rotation angles using détente presets could also be obtained using the same inhale-exhale countdown. The typical subject set/hold time for imaging was about one minute following subject alignment. Typical first use education and "subject fitting" required approximately 10-15 minutes. Subsequent visits required a short review with completion in less than five minutes. All images were obtained with the eyes open, There was an advantage to closed eye imaging during movement for the chair for rotation angle imaging.

Contact with head touch points, preset to individual parameters, was maintained by the subject and allowed undistorted facial imaging of the face, jaw, and neck regions. The touch points were defined by the height above the seated position, distance from vertical orientation, occipital touch point location, top frontal bone touch point position, and neck locator position. These defined parameters were linked with a specific direction-of-gaze parallax bar height. The device and alignment process minimized variation in the four axes of co-location: depth, tilt, rotation, and twist. Re-alignment parameters of the device were verified between operators to within 0.1 to $0.2 \mathrm{~mm}$ for all adjustments.

\subsection{Components and Construction}

The device described included a direction-of-gaze parallax bar system and a subject chair with headrest.[5] The direction-of-gaze parallax bar system (Fig. 3. and 4.) consisted of a tripod mount base, a modified enlarger stand mounted on a specialty base interface plate, and an extruded aluminum support structure holding the parallax bars, 2D camera, 3D projectors/sensors, and lighting. The entire system could be disassembled for shipping followed by reassembly at $3^{\text {rd }}$ party clinical testing laboratories. Reproduction of equivalent overlaid images, prior to and following shipping and reassembly, was not required.

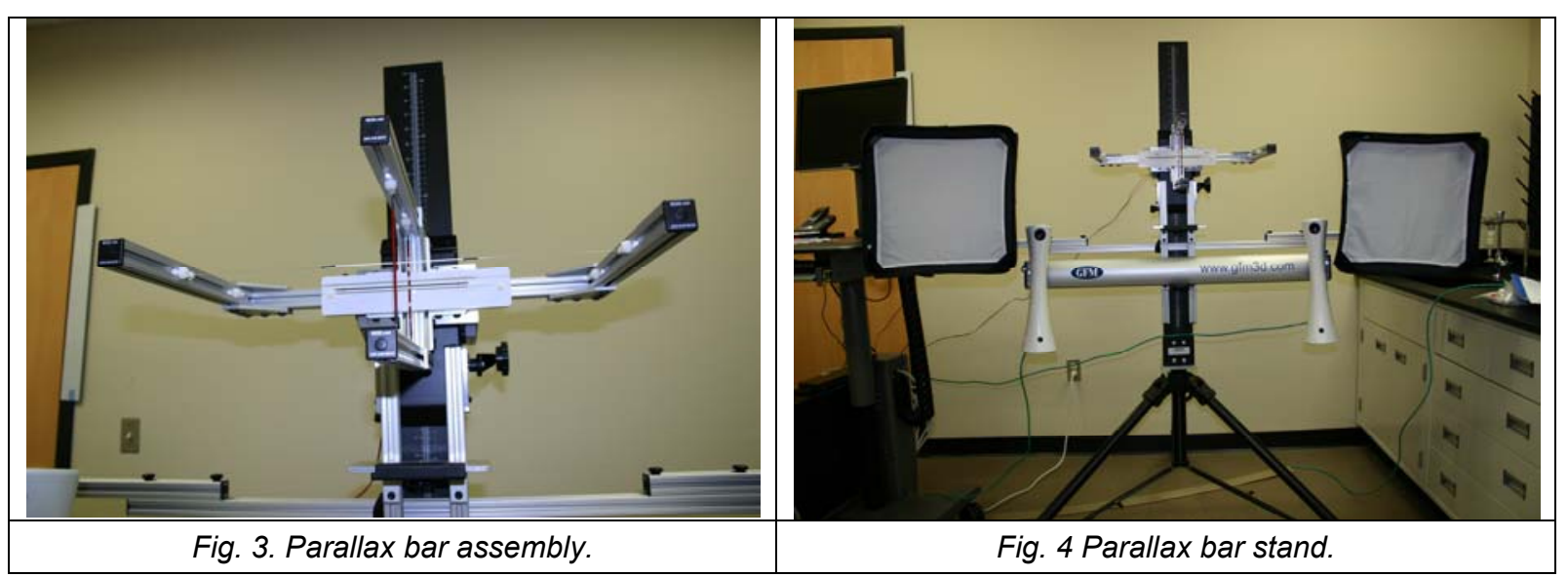




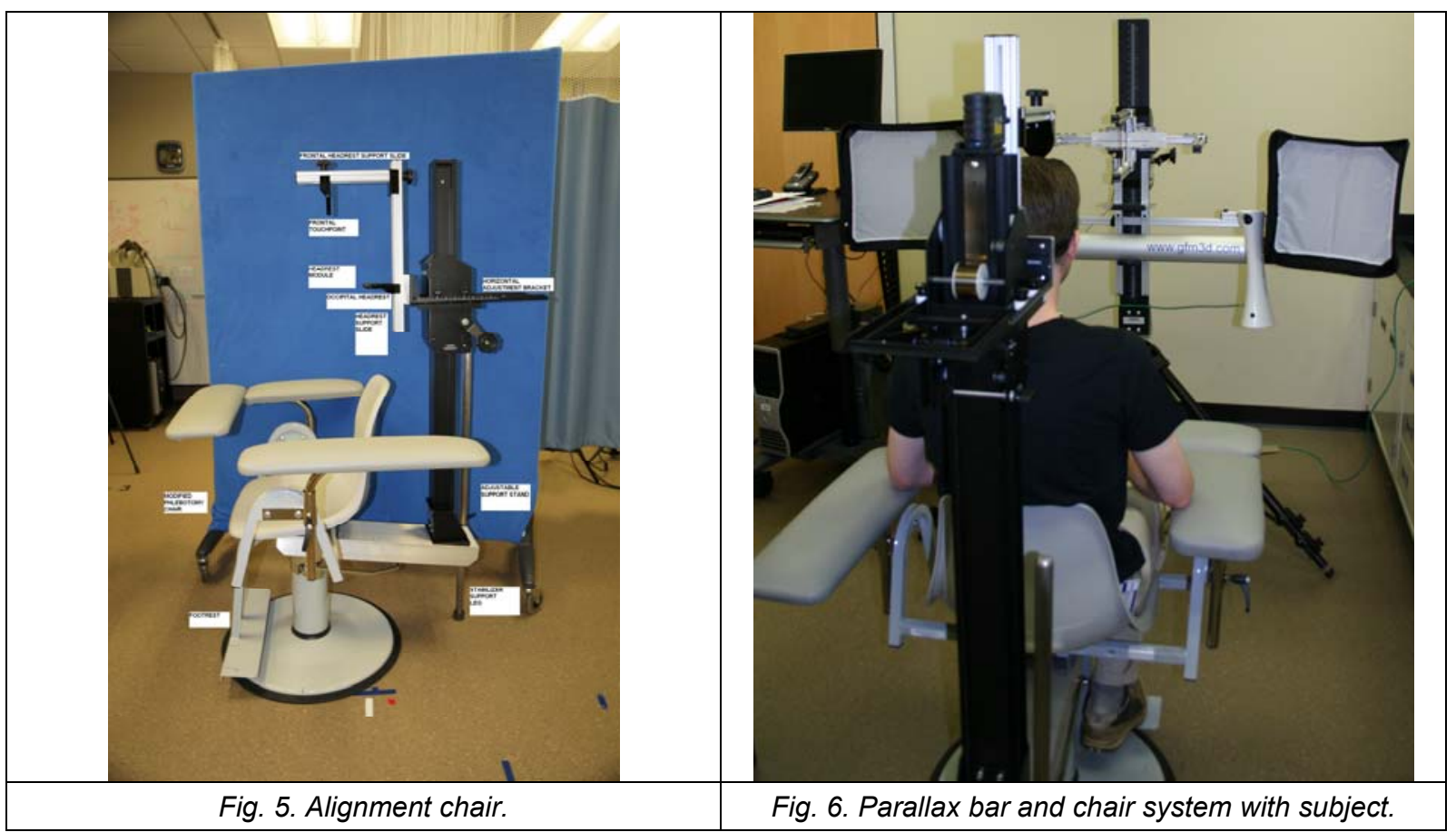

The parallax bar frame (Fig. 3.) was constructed of extruded aluminum bars (McMaster Carr Aluminum T-Slotted Framing, Santa Fe Springs, CA, USA) and was designed and manufactured within the Rapid Prototyping Department of Durables, Amway R\&D, Ada, MI, USA. The parallax bar supports were constructed from the same extruded aluminum. The parallax bars were constructed of fine polymer wire with a colored polymer coating, and held in adjustable tension holders within the extruded aluminum frame. Bar color was selected for contrast and ease of viewing for parallax identification (overlay and centering).

The tripod base (Fig. 4.) supported the adjustable bracket holding the parallax bars and imaging equipment. The tripod (Manfrotto 161MK2B Tripod, Bassano del Grappa, Italy) was selected for weight strength and rigid stability.

An enlarger copy stand (Fig. 4.) supported the parallax bars and imaging equipment. A second stand, mounted on the chair assembly (Fig. 5.), supported the head alignment brackets The stands (Beseler CS Digital/Photo-Video Copy Stand-44" (BS8410), Stroudsburg, PA, USA) were chosen for range of height adjustability, rigidity, weight capacity and modifiable brackets.

The 2D - 3D - lighting support was constructed from extruded aluminum and routed plate aluminum. The 2D support had a custom camera mount form fit to the camera for ease of removal and realignment. The 3D mount was hinged and contained a location for inserting custom angle solid supports to permit up-angled 3D imaging. The 10-degree mount was used for this study. All custom materials were designed and manufacture within the Rapid Prototyping Department of Durables, Amway R\&D, Ada, MI, USA.

The chair (Fig. 5. and 6.) was a custom modified phlebotomy chair (Medicus Health Hydraulic Phlebotomy Chair (2311M1-Dove), Kentwood, MI, USA). The rotational angle (détente) ring and aluminum support extension was added to the chair to permit preset angles for the chair rotation. The main support stand for the head rest was the enlarger copy stand previously described. The adjustable head, neck and shoulder brackets were custom made from aluminum plate or extruded aluminum. The head touch point surfaces were constructed from nylon and coated with automotive paint. For non-rotational angle studies, a rear support stand was utilized and was affixed to the chair aluminum support extension for additional stability. The floor template for device orientation was laid integrally to the parallax bar support stand and chair, and was able to be disassembled into sections. All custom components for the phlebotomy chair and head rest assemblies were produced by Rapid Prototyping Department of Durables, Amway R\&D, Ada, MI, USA. 
Two-dimensional digital images were obtained using a Canon 5D Mark II with a Canon 50mm f/1.4 lens, Tokyo, Japan. Three-dimensional renderings were obtained using a GFM FaceScan3D system with two-sensors (GFMesstechnik GmbH, Berlin, Germany)[2]. Collection and analysis software used was Facexenios 4.3.1 for GFM Facescan 3D. Off-line evaluations and calculations were performed using 3DViewer V.1.540 (3D-Shape $\mathrm{GmbH}$, Erlangen, Germany). Additional calculations were performed using Microsoft Excel.

The combined parallax bar and chair system (Fig. 6.) was capable of disassembly for shipping and storage in impact resistant polymer shipping cases.

\subsection{Subjects}

Subjects imaged during device development consisted of healthy volunteers from among laboratory employees. There were no special considerations for the selection of the volunteers except for interest and available time. Manikins were used for general testing and served as repeatability standards. Extended studies for image overlay were conducted from a recruited panel of 40 subjects willing to participate in the study and to appear at assigned analysis times and dates. Three age ranges were selected: $20-25$ years ( 2 groups, 10 subjects each), $40-45$ years (10 subjects), and $60+$ years (10 subjects). All subject had a BMI between 21 and 26 . Subjects that provided insufficient projector light reflection were eliminated from the 3D overlay portion of the study. Subjects that were unable to focus appropriately were eliminated from the 3D portion of the study. Image overlay comparisons were performed between initial and subsequent imaging sessions. Each of the subjects provided written and signed informed consent forms prior to participation in these studies.

\subsection{Analysis}

The skin surface was analyzed using a Primos Facescan3D with two sensors (GFMessentechnik $\mathrm{GmbH}$ ). This instrument is based on the principal of digital stripe projection. Digital stripes are projected onto the facial skin surface and images are collected on a camera CCD sensor during the projection. Small variations occurring due to non-even skin surfaces are detected by the camera sensor as a deflection and registered. The deflections are measured quantitatively and recreated as a 3D rendering from which quantitative measurements such as surface area (through triangulation), volume (difference in triangulation surfaces) and perimeters/cross-sections and distances may be obtained. Addition of differential color effects can provide improved qualitative differential viewing to the renderings. The imaging system employed two camera projection/sensors mounted horizontally, which provided overlapping imaging regions of the face and neck. Computer integration of the data sets rendered ear-to-ear 3D images of the face.

\section{Test/Data}

\subsection{Computer overlay of 3D Images}

Primary and fine co-location (overlay) was performed through positioning of the subject using the parallax bar and chair system. Final computer adjustment of the overlaid images was obtained through three separate analysis steps comparing images gathered during separate subject visits:

1) elimination of data outside the facial regions of interest

2) general facial registration of the two images

3) set point reference selection on the reference image (e.g. bottom center of earlobes, nose tip, center of forehead, center of sternal notch) and localized stable surface reference area selection (e.g. ears, forehead, length and end of nose, expanded sternal notch area).

Images obtained for 3D analysis were similar to those depicted in Fig. 7., Fig. 8., and Fig. 9. These 3D images were utilized for cross sectional analysis (Fig. 10.) and comparative before-and-after visualization. The $3 \mathrm{D}$ image, or rendering, enabled viewing at multiple angels for inspection. The differences between two aligned 3D single images at two subject session were compared using the steps noted above, and a topological color image similar to Fig. 11. and Fig. 12., was produced for each subject. These images and differential values were used to determine the surface area and volume measurements in selected regions. Comparative overlaid cross sections were also obtained. 


\subsection{Computer cross-sections}

Co-located (overlaid) renderings were compared for differential measurement and topology differences. Parallel vertical cross-sections were obtained at the profile, proximal to the left/right nostrils, center of left/right eyes, and outer edges of left/right eyes (Fig. 7.). Parallel horizontal crosssections were obtained at the zygomatic bone, nose tip, top of philtrum, center of philtrum, mouth, concave (centroid) of chin, and just below chin (Fig. 8.). Parallel diagonal cross-sections were obtained through the jaw, and at two centimeter internals below the jaw (Fig. 9.) Cross-sections were obtained from single and overlaid 3D images. Single 3D images, from study subjects in the same age bracket were centered and cross sections were overlaid (Fig. 10.).

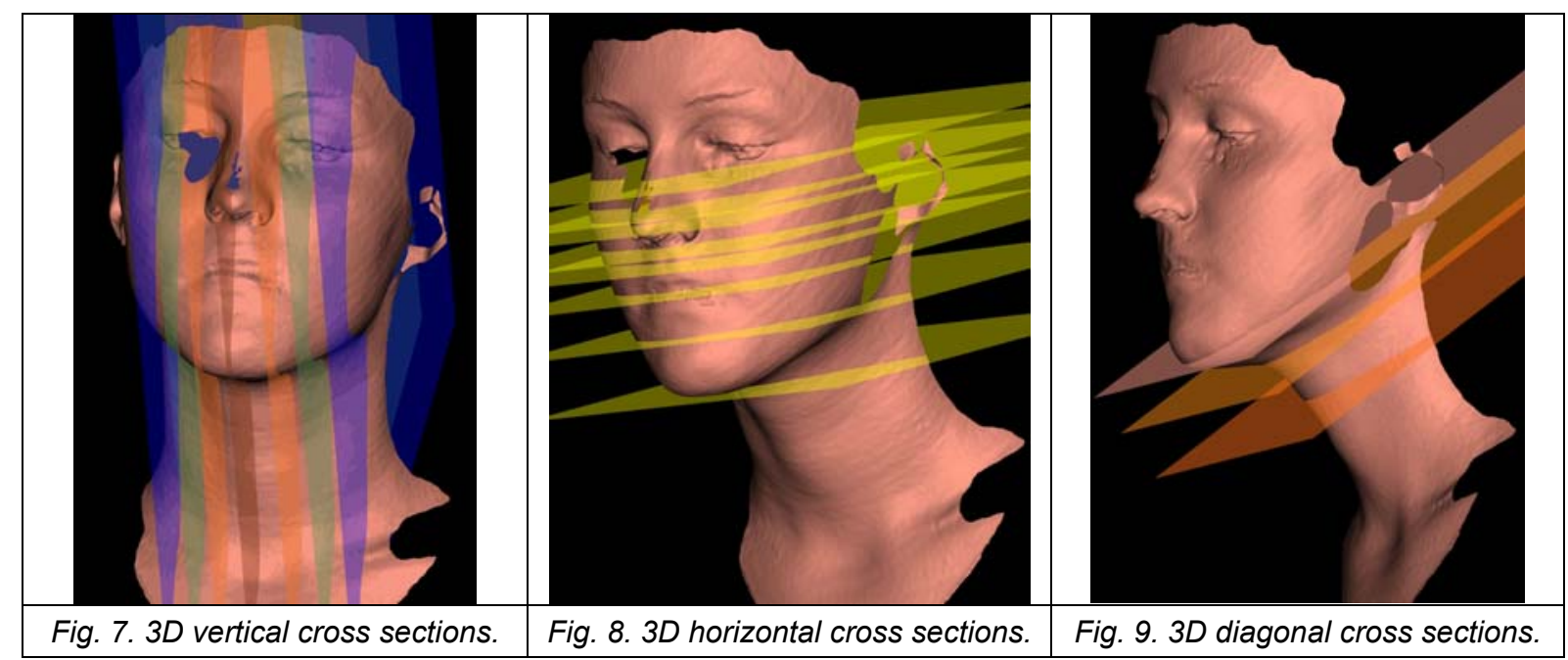

Diagonal Jowl Cross Section $(\mathrm{Age}=60+$ )

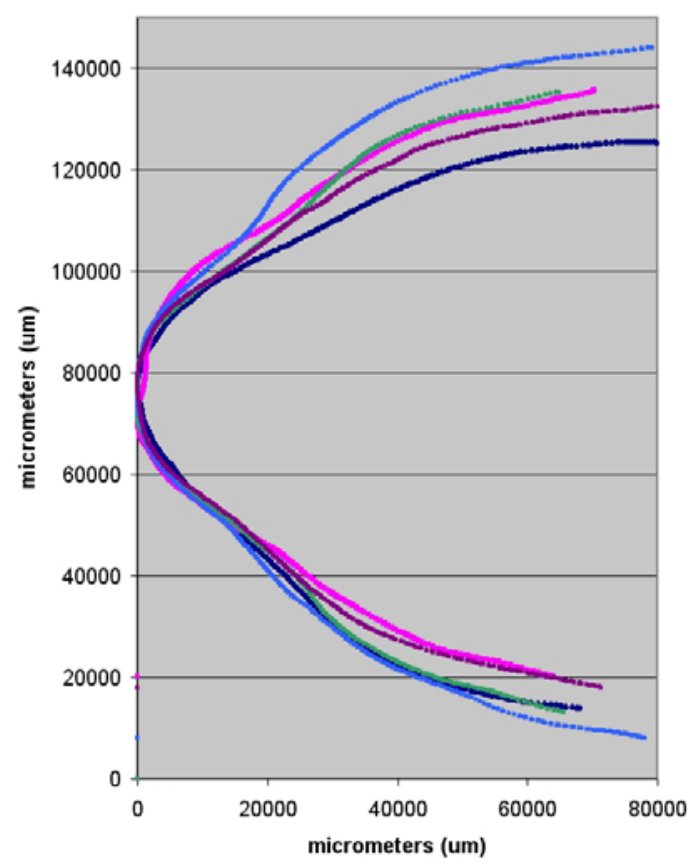

Fig.10. Jowl cross section (60+) overlay.

Two dimensional digital images were obtained through chair rotation immediately following 3D image acquisition at $0^{\circ},+45^{\circ},\left(-45^{\circ}\right),+90^{\circ},\left(-90^{\circ}\right)$ to full face. 


\section{Results}

\subsection{Alignment}

The reference objects, such as a manikin head/neck, provided repeatable differential measurements between imaging sessions. Spatial variations of less than 100 um were typically observed over the entire facial and neck areas. The manikin head was strapped into the head rest during the image session (Fig. 11.). The 100 um difference observed from sequential samples provided what was considered best fit data compared to what might be achieved for any human subject. A slightly repositioned manikin head was considered to provide a best fit variation equivalent for repeat visits of human subjects during stages of clinical analysis. This variation remained near 100 um (Fig. 12.).

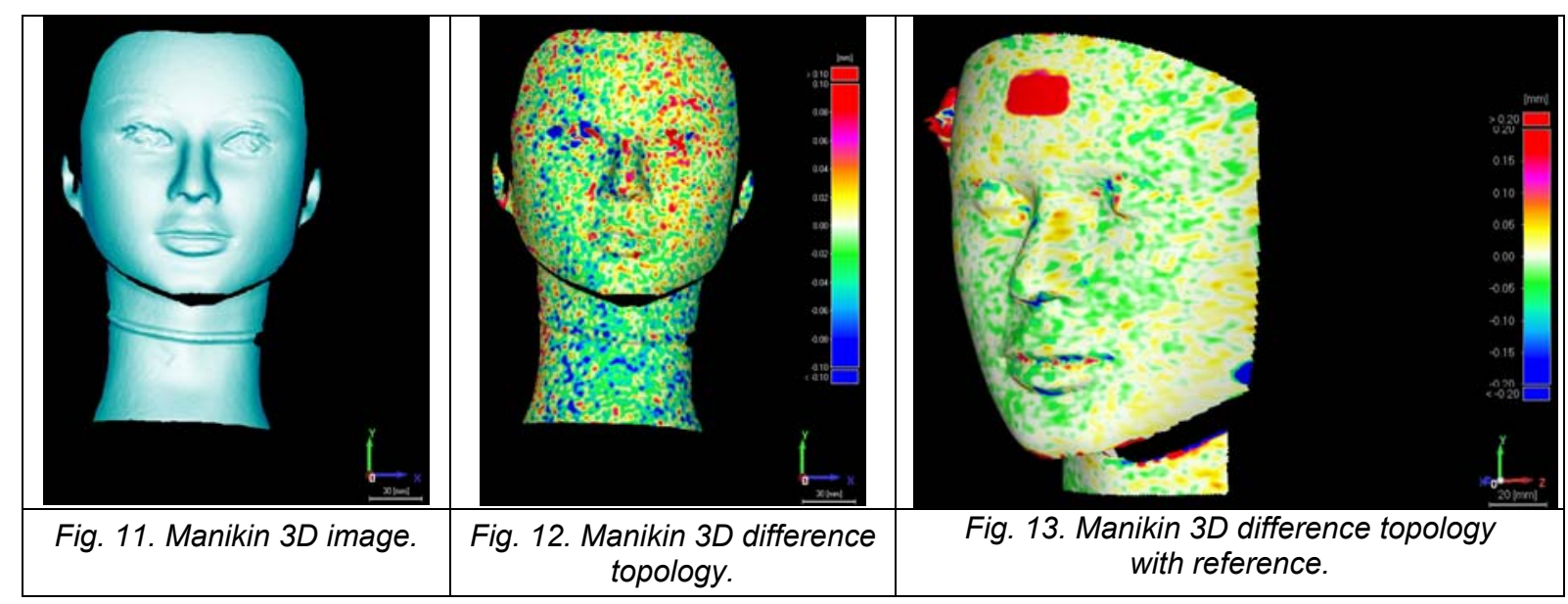

Volume measurement consistency was evaluated using standardized reference application stickers. The stickers were sufficiently small and pliable as to be applied for intermittent reference checks of overlaid image analysis studies. The statistical summary of ten replicate reference sticker applications was obtained on a manikin (Fig. 13.), and compared to the known volume obtained from micrometer readings. (actual volume $=0.0624 \mathrm{~cm}^{3}$; $\max$ volume $0.06429 \mathrm{~cm}^{3}$; $\min$ volume 0.06113 $\mathrm{cm}^{3} ; 3 \mathrm{D}$ volume $=0.0629 \pm .0017 \mathrm{~cm}^{3}$ ). This same technique was utilized to determine skin plumping and puffy-eye reduction for before-and-after cosmetic application trials.

Human alignment was not expected to match that of the manikin. Differential topology over the face in the range of $\pm 1 \mathrm{~mm}$ deflection was desired. Except for the immediate eye and mouth area, most surfaces of subject faces overlaid with a variation of 500 um (Fig. 14. through Fig. 16). Typical variations encountered were in the sub-ocular contour area associated with eye blinking and at the lip and mouth edges due to closure variations. Images obtained using the countdown exhalation process provided uniform topology, but images obtained during inhalation created approximately 250um variations proximal to the nasal area. Swallowing was noted and easily observed, so during the countdown subjects were instructed to not swallow. As an example of variation, a thin retail adhesive bandage was applied to a subject's neck and the subject imaged (Fig. 14. and Fig. 15.). The subject left the room, removed the bandage, and was re-imaged. A false color, 3D mesh reconstruction topology result is presented (Fig. 16.). The bandage was easily detected and measurable on the neck. Even the section of pulled skin near the left edge of the bandage was observed. (The bandaged image was the reference image, so the topology indicated negative surface change. In Fig. 12., the manikin without the sticker was the reference image, so the sticker topology was positive red.) 


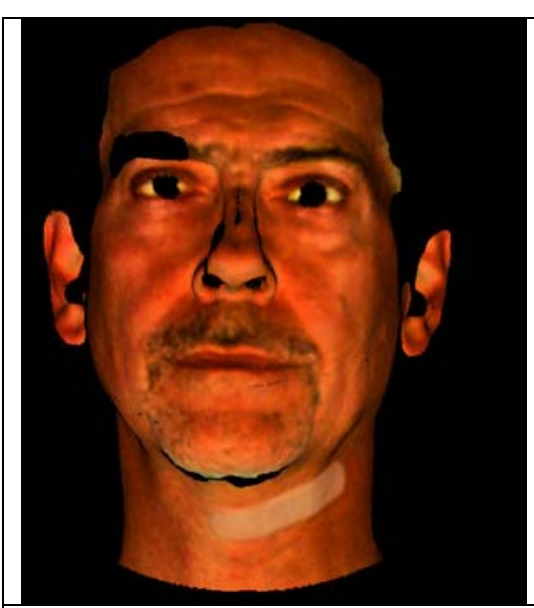

Fig. 14. False color 3D image with bandage.

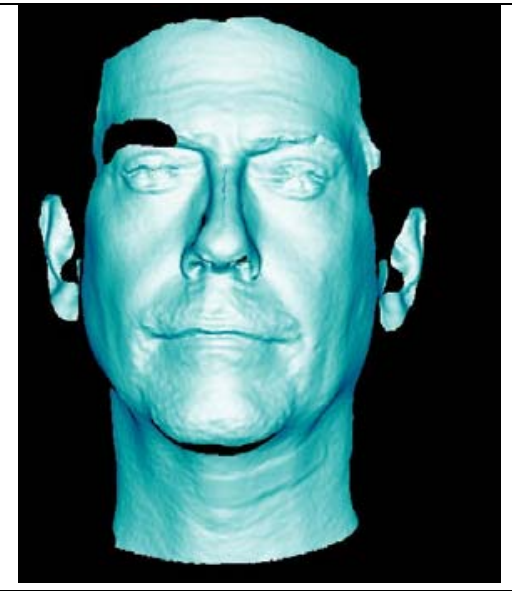

Fig. 15. 3D Image with bandage.

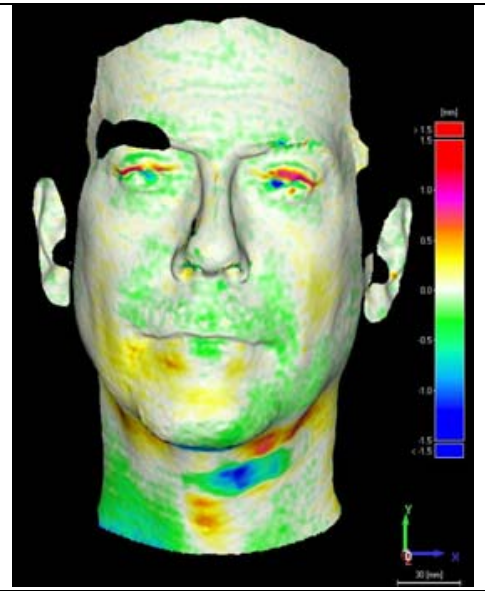

Fig. 16. 3D difference topology with and without bandage.

\section{Conclusions and Discussion}

Co-location of the subject for subsequent imaging was paramount for high quality image overlay. With current subject alignment equipment and procedures, the direction of the subject face was highly variable in depth to camera, tilt, rotation, yaw, and twist. These image variations occurred even when the optical tables and chairs were adapted for subject comfort. Pressure points on the forehead, and compression of the chin resulted in extreme deformations of the chin, mouth, lips and cheek areas. Chin mounts occluded the lower portion of the face and the entire neck. Extended acquisition times resulted in subject relaxation during image acquisition and many (2D) images with acceptable overlay variation differed significantly when compared in 3D alignment.

The integrated positioning device eliminated these encumbrances, and reduced movement and alignment effects. The removal of camera-visible facial supports allows the subject to be imaged in a natural pose thus reducing image and alignment artifacts. The parallax bar concept for orientation and direction-of-gaze enabled the subjects to perform the bulk of the realignment themselves. The recording of all unique chair and parallax bar parameters for each subject enabled individualization of the entire alignment system. Comparison between operators of device parameters indicated that all set-point values could be reproduced within $\pm 0.1-0.2 \mathrm{~mm}$.

The parallax alignment concept was not immediately clear to all subjects. The capacity for realignment was dependent on the system education and preparation of the subjects prior to imaging. It was apparent when subjects did not understand the concept of parallax and overlay. This was observed by large realignment movements of the head. Unprepared study participants provided significantly different (poorer) result images than trained participants. Training significantly reduced time for subject reorientation at subsequent visits.

Inherent subject variations observed included: slight eyebrow change and blinking (Fig. 17.), slight head twist, lip and jaw closure (Fig. 18.), and inhalation breathing variations (Fig. 19.). Breathing variations, observed as volume changes proximal to the nose, were greatly reduced by the breathing countdown process prior to image capture. Lip closure variations were minor, but were not entirely eliminated. Neck twist variation at the body intersection was of most concern in the methodology. 


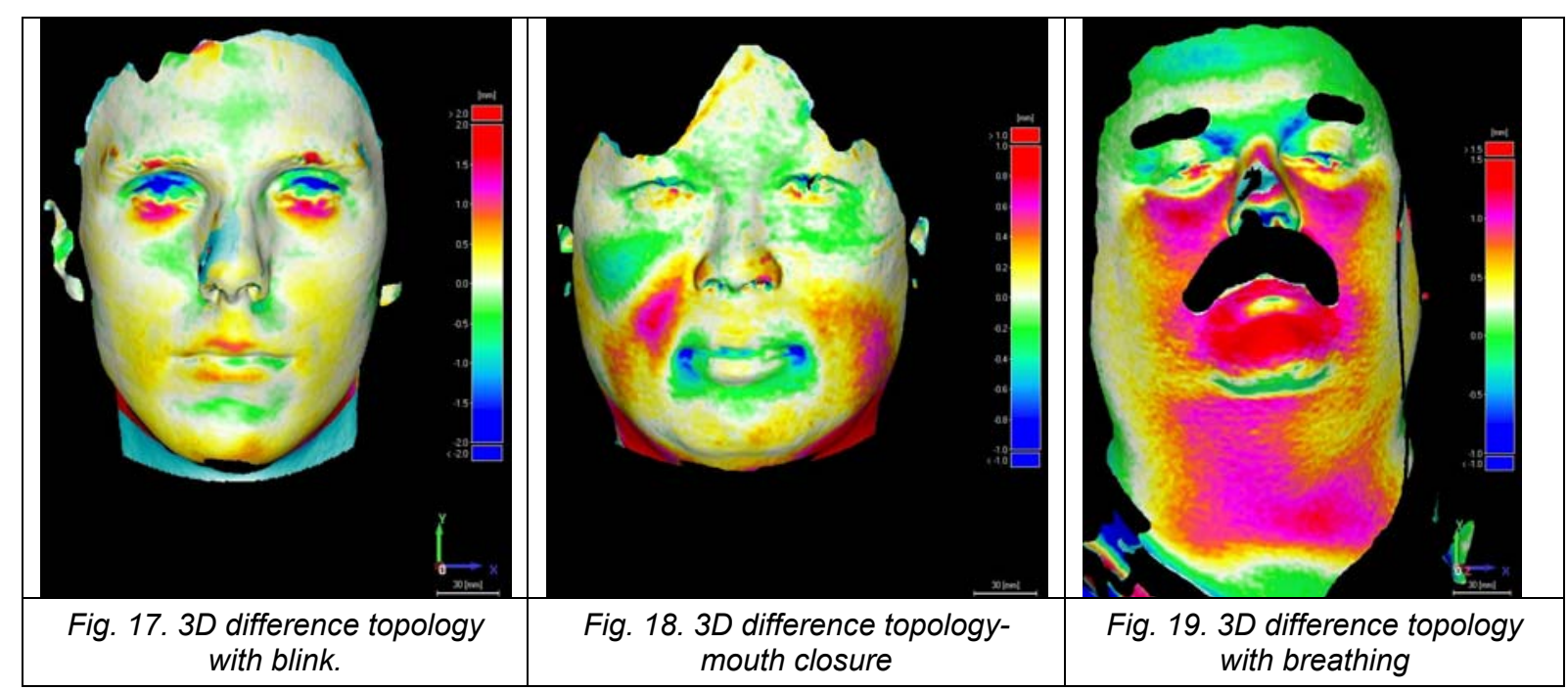

Subjects exhibited neck twist as the greatest and most frequent variation. Many $2 \mathrm{D}$ images that appeared closely aligned were found to have more than the desired topology differences in neck twist variation when viewed through 3D overlay. Extreme care in seating and subject placement was found to reduce but not eliminate this issue. To alleviate most of the neck twist, a post alignment adjustable (recorded parameter) v-shaped neck bar for touch location only was included within the headrest bracket. Our results indicated the bar improved subsequent neck alignment.

In summary, the study results obtained using the parallax bar and chair alignment system was a significant improvement over standard alignment techniques. Subject training, education, and understanding of the alignment process was important. The system design and study results demonstrated a means of obtaining fine overlay of facial areas for imaging while relying on subject based primary alignment. The alignment and analysis protocols provided a good method for overlaying $2 \mathrm{D}$ and $3 \mathrm{D}$ images without deformation or encumbrance at the face or neck. The repeatability of the alignment and analysis protocols indicated the potential for $3 \mathrm{D}$ overlay image analysis for cosmetic imaging and for overlay visualization in cosmetic surgery.

\section{References}

1. Face scanning system "Realscan 3D", 3DDigital Corporation (accessed 2012): www.3ddigitalcorp.com

2. Face scanning system "FaceScan" (accessed 2012): www.gfm3d.com

3. Whitmer, K., Barford, B., Turner, M., Sullivan, D., and Sommers, M., (2011) "Digital image analysis of facial erythema over time in persons with varied skin pigmentation", Skin Research and Technology, Vol.17, No.3. pp.348-352.

4. Head positioning system "HeadScan", Orion Concept (accessed 2012): www.orion-concept.com

5. Roth, R., DePauw, M.,, and Hepner, A., "Devices for Positioning Subjects", U.S. Provisional Patent No. 61/635,592, April 19, 2012. 